\title{
Detergent-induced aggregation of an amyloidogenic intrinsically disordered protein
}

\author{
SHRUTI ARYA $^{\mathrm{a}, \mathrm{b}, \mathrm{d}, *}$, PRIYANKA DOGRA ${ }^{\mathrm{a}, \mathrm{b}}$, NEHA JAIN $^{\mathrm{c}, \mathrm{e}}$ \\ and SAMRAT MUKHOPADHYAY ${ }^{\mathrm{a}, \mathrm{b}, \mathrm{c}, * \text { (B) }}$ \\ ${ }^{\mathrm{a} C e n t r e}$ for Protein Science, Design and Engineering, Indian Institute of Science Education and Research (IISER) \\ Mohali, Mohali, Punjab, India \\ ${ }^{b}$ Department of Chemical Sciences, Indian Institute of Science Education and Research (IISER) Mohali, Mohali, \\ Punjab, India \\ ${ }^{\mathrm{c}}$ Department of Biological Sciences, Indian Institute of Science Education and Research (IISER) Mohali, Mohali, \\ Punjab, India \\ ${ }^{\mathrm{d}}$ Present address: Department of Chemistry and Biochemistry, University of California Santa Barbara, Santa \\ Barbara, CA, USA \\ ePresent address: Department of Molecular, Cellular and Developmental Biology, University of Michigan, Ann \\ Arbor, MI, USA \\ E-mail: shrutiarya.chem@gmail.com; mukhopadhyay@iisermohali.ac.in
}

MS received 8 August 2017; revised 22 September 2017; accepted 2 October 2017; published online 2 November 2017

\begin{abstract}
Intrinsically disordered proteins (IDPs) belong to an important class of proteins that do not fold up spontaneously. The conformational flexibility of IDPs allows them to adopt a wide range of conformations depending upon their biochemical environment. Many IDPs undergo profound conformational conversion that is often coupled to amyloid aggregation in the presence of negatively charged lipid membranes. Here, we show the effect of a well-known anionic lipid mimetic, sodium dodecyl sulfate (SDS), on the aggregation mechanism of a model amyloidogenic IDP, namely, bovine $\kappa$-casein. In the absence of SDS, the aggregation kinetics of reduced and carboxymethylated (RCM) $\kappa$-casein followed a nucleation dependent polymerization model that comprises both lag- and assembly phases. On the contrary, in the presence of sub-micellar concentration of SDS, the aggregation kinetics did not exhibit a lag phase and appears to follow a non-nucleation pathway. Additionally, the morphologies of the aggregates formed in the absence and presence of SDS were found to be different. In the absence of SDS, $\kappa$-casein aggregation proceeded to typical amyloid fibrils, whereas, in the presence of SDS, the aggregation yielded large oligomers. Our results provide important molecular insights into the aggregation mechanism that can be utilized for the designing of novel protein/amyloid based nanomaterials with desired properties.
\end{abstract}

Keywords. Amyloid formation; fluorescence spectroscopy; intrinsically disordered proteins; protein aggregation.

\section{Introduction}

Protein aggregation resulting in amyloid formation is associated with a variety of deadly human diseases. ${ }^{1-7}$ Recently it has been realized that highly ordered amyloid aggregates can also serve as promising template for the fabrication of nano-structures for a wide variety of applications such as in bio-nanotechnology ${ }^{8-11}$ and

\footnotetext{
*For correspondence

Dedicated to Professor Uday Maitra on the occasion of his 60th birth anniversary.
}

food processing industry. ${ }^{12,13}$ Typically, protein aggregation is favored under the conditions that lower the stability of the native form. ${ }^{14}$ An enormous effort has been made to elucidate the mechanism of amyloid formation. ${ }^{15,16}$ However, the precise mechanism of amyloid formation still remains elusive due to the inherent complexities associated with the process. One of the important factors that can trigger protein aggregation is the presence of surfaces with certain physicochemical features that favor aggregation by profoundly influencing the stability of the native state of a protein. ${ }^{17,18}$ The recruitment of proteins or peptides by synthetic or biological surfaces can result in the conformational 
transition of the native (functional) state to the nonnative, aggregation prone (non-functional/pathological) state. ${ }^{17,18}$ Therefore, there is a growing interest in investigating the physicochemical characteristics of interaction between protein and synthetic or natural surfaces, and aggregation of proteins on these surfaces such as lipid membranes. The protein-membrane interactions are believed to play a key role in protein misfolding. ${ }^{19-21}$ In fact, many of the pathological amyloid deposits have been found to interact with membranes thereby causing membrane disruption ${ }^{22-30}$ and many of these pathological amyloids are often formed by intrinsically disordered proteins (IDPs), ${ }^{22,24,25}$ a class of proteins that lack the ability to undergo autonomous folding. ${ }^{31,32}$ Amphiphilic sodium dodecyl sulfate (SDS) provides a membrane mimetic environment and hence have been extensively used for studying the role of membrane in amyloid formation by various proteins, including IDPs. ${ }^{33-36}$

In this study, we have investigated the influence of SDS as a membrane mimetic on the aggregation and fibrillation of a model amyloidogenic IDP, namely, bovine $\kappa$-casein. $\kappa$-casein is a milk protein and is a member of a protein family called caseins. Caseins belong to the functional class of IDPs known as scavengers. ${ }^{37}$ They are the major protein elements of mammalian milk and play an important role in the cellular uptake of calcium and phosphate ions. ${ }^{38,39}$ Additionally, caseins are well recognized for their capability to act as molecular chaperones. ${ }^{40-44}$ The chaperone-like activity of $\alpha_{\mathrm{s} 1}-$ and $\beta$-casein also has considerable physiological advantages in preventing the amyloid formation from two amyloidogenic caseins, $\alpha_{\mathrm{s} 2}-$ and $\kappa$-casein, under physiological conditions. ${ }^{45-47}$ The amyloid formation by $\kappa$-casein is linked with mammary cancer. ${ }^{48,49}$ Since $\kappa$-casein is a milk protein, therefore, in order to understand the process of amyloid formation from $\kappa$-casein, it is important to consider the influence of various components of milk on amyloid formation. One of the important components of milk is lipids that comprise approximately $4 \%$ $\mathrm{w} / \mathrm{v}$ of milk. ${ }^{50}$ In addition, the membrane of mammary epithelial cells is composed of phospholipid bilayer. ${ }^{51}$ Therefore, studying the effect of lipids or lipid mimetics on $\kappa$-casein is of physiological relevance to elucidate the mechanism of $\kappa$-casein aggregation. Here, we have used SDS, an anionic detergent, to mimic membrane environment in $\mathrm{\kappa}$-casein fibrillation. In vitro protein aggregation studies have revealed that the kinetics of fibrillation is often altered in the presence of lipids/detergents. ${ }^{52,53}$ In many cases, they accelerate the fibrillation process. ${ }^{33,34}$ However, there are cases where they can have inhibitory effect on fibrillation. ${ }^{54,55}$ Here we show that SDS alters the pathway and morphology of $\kappa$-casein aggregation.

\section{Experimental}

\subsection{Materials}

$\kappa$-casein (from bovine milk), dithiothreitol (DTT), iodoacetic acid, guanidinium chloride $(\mathrm{GdmCl})$, Tris $\mathrm{HCl}$, sodium chloride $(\mathrm{NaCl})$, sodium dodecyl sulfate (SDS), 8-anilino-1naphthalenesulfonic acid ammonium salt (ANS) and sodium hydrogen phosphate (monobasic) were procured from Sigma (St. Louis, MO) and used as received. 5-((((2-iodoacetyl) amino)ethyl)amino) naphthalene-1-sulfonic acid (IAEDANS) was purchased from Molecular Probes, Invitrogen Inc. All solutions were prepared in Milli-Q water. The $\mathrm{pH}$ of the buffers was adjusted using a Metrohm $\mathrm{pH}$ meter at $\sim 25^{\circ} \mathrm{C}$.

\subsection{Circular dichroism (CD) measurements}

The CD measurements were carried out on Chirascan Spectrophotometer (Applied Photophysics, UK) using a $1 \mathrm{~mm}$ path length quartz cell, and the spectra obtained were corrected for buffer background signal. The buffer corrected spectra were then smoothened using Pro Data software. All the measurements were made at room temperature.

\subsection{Steady-state fluorescence measurements}

For all the experiments, except for the data shown in Figure 1A, $\kappa$-casein was reduced and carboxymethylated (RCM) using DTT and iodoacetic acid using the protocol described previously ${ }^{56,57}$ and the stock solution of $\kappa$-casein was stored under denatured condition $(6 \mathrm{M} \mathrm{GdmCl}$ in $\mathrm{pH} 7,50 \mathrm{mM}$ phosphate buffer). For Trp steady-state fluorescence and CD measurements, the protein stock was diluted into the native buffer (pH 7.2, $50 \mathrm{mM}$ phosphate buffer) to obtain a final concentration of $20 \mu \mathrm{M}$. For SDS binding experiments, a fresh stock of $10 \mathrm{mM}$ SDS was prepared in Milli-Q water and was used for making further dilutions. For AEDANS fluorescence measurements, reduced and denatured $\kappa$-casein was labeled with a 50 molar excess of IAEDANS under denatured condition (6 M GdmCl in pH 7.6, $100 \mathrm{mM}$ Tris buffer, $2 \mathrm{~h}$ at $37^{\circ} \mathrm{C}$ ). The labeled protein was then passed through a PD-10 column to remove excess dye and was further concentrated using AMICON ultra (3 kDa cutoff; from Millipore). The concentration of the labeled protein was estimated using $\varepsilon_{337 \mathrm{~nm}}=6100 \mathrm{M}^{-1} \mathrm{~cm}^{-1}$ for AEDANS. ${ }^{58}$ The AEDANS labeled $\mathrm{\kappa}$-casein was stored under denatured condition (6 M $\mathrm{GdmCl}$ in $\mathrm{pH} 7,50 \mathrm{mM}$ phosphate buffer).

All the steady-state fluorescence measurements were carried out on Fluoromax-4 (Horiba Jobin Yvon, NJ) at $\sim 25^{\circ} \mathrm{C}$. The samples were excited at $295 \mathrm{~nm}$ (Trp), $450 \mathrm{~nm}$ (ThT) and $375 \mathrm{~nm}$ (ANS and AEDANS). The steady-state fluorescence anisotropies were measured at $350 \mathrm{~nm}$ (Trp) and $480 \mathrm{~nm}$ (ANS and AEDANS). The steady-state fluorescence anisotropy $\left(\mathrm{r}_{\mathrm{ss}}\right)$ is given by the following relationship:

$r_{s s}=\frac{I_{\|}-I_{\perp} G}{I_{\|}+2 I_{\perp} G}$ 
where $I_{\|}$and $I_{\perp}$ are fluorescence intensities collected using parallel and perpendicular geometry, respectively. For Trp fluorescence kinetics measurements, $\kappa$-casein in native buffer was taken as baseline and then SDS (100 $\mu \mathrm{M})$ was added to this solution. The SDS solution was manual mixed and the time of mixing was typically 5-10 seconds. For monitoring ThT and ANS fluorescence kinetics upon addition of SDS $(100 \mu \mathrm{M})$; ThT $(20 \mu \mathrm{M})$ and ANS $(20 \mu \mathrm{M})$, respectively were already present in the buffer containing $\kappa$-casein $(20 \mu \mathrm{M})$. All the measurements were made at room temperature.

\subsection{Aggregation reaction}

The aggregation reaction was initiated using $200 \mu \mathrm{M}$ of RCM $\kappa$-casein in $50 \mathrm{mM}$ phosphate buffer $(\mathrm{pH} 7.2)$. The reaction mixture was continuously stirred at a speed of $300 \mathrm{rpm}$ and the temperature was maintained at $37^{\circ} \mathrm{C}$. The aliquots $(10 \mu \mathrm{M})$ were taken out at several time points and Trp fluorescence was measured. For measuring ThT and ANS fluorescence, $10 \mu \mathrm{M}$ of ThT and ANS, respectively were added to the aliquots taken out from the aggregation mixture. The final protein concentration for measurement was $10 \mu \mathrm{M}$. All the final dilutions were made in $50 \mathrm{mM}$ phosphate buffer ( $\mathrm{pH}$ 7.2). For carrying out aggregation reaction with AEDANS labeled $\kappa$-casein, $20 \mu \mathrm{M}$ of AEDANS labeled $\kappa$-casein was added to $180 \mu \mathrm{M}$ of RCM $\kappa$-casein. The aliquots were taken out at different time points and the AEDANS fluorescence was measured without any further dilution. The parameters used for fluorescence measurements are mentioned above. The fluorescence data at different points were then plotted and fitted with the following equation described previously for nucleation-dependent polymerization. 59

$y=y 0+\frac{a}{1+\exp \left(-\frac{x-x 0}{b}\right)}$

where $y$ is the fluorescence at time $x, y_{0}$ is the initial fluorescence value, $x_{0}$ is the time when fluorescence reaches $50 \%$ of its maximum value, and $a$ is the maximum fluorescence at stationary phase. The lag time is given by $\left(x_{0}-2 b\right)$.

\subsection{Time-resolved fluorescence measurements}

The fluorescence lifetime data were acquired using a timecorrelated single photon counting (TCSPC) setup (Fluorocube, Horiba Jobin Yvon, NJ). All the measurements were made at room temperature. The peak count was fixed to 10,000 and the excitation and emission polarizers were oriented at the magic angle $\left(54.7^{\circ}\right)$. For time-resolved fluorescence anisotropy measurements, the peak difference was 10,000 counts and the orientation of the emission polarizer was $0^{\circ}$ and $90^{\circ}$ with respect to the excitation polarizer for parallel fluorescence intensities $\left(I_{\|}\right)$and perpendicular fluorescence intensities $\left(I_{\perp}\right)$, respectively. The emission monochromator for ANS was fixed at $480 \mathrm{~nm}$ with a bandpass of $12 \mathrm{~nm}$ and 375 $\mathrm{nm}$ laser diode was used as excitation source. The instrument response function (IRF) was collected using Ludox (colloidal silica). The width (FWHM) of IRF was $\sim 250$ ps. The fluorescence intensity decay curves were deconvoluted taking IRF into account using

$$
I(t)=\sum_{i} \alpha_{i} e^{-t / \tau_{i}}
$$

\subsection{Atomic force microscopy (AFM) imaging}

AFM images were acquired on an Innova atomic force microscope (Bruker). The AFM was operated in tapping mode. For imaging, the silicon nitride cantilever probe with radius $\sim 8 \mathrm{~nm}$ was used. The samples (typically diluted 10002000 fold with aggregation reaction buffer filtered through a $0.22 \mu \mathrm{m}$ syringe filter) were deposited on a freshly cleaved muscovite mica (Grade V-4 mica from SPI, PA). The mica surface was incubated with the sample for 15-20 min before washing it with filtered Milli-Q water and dried under a gentle stream of nitrogen gas. The images were collected in NanoDrive (v8.03) software at a resolution of 1024 x 1024 pixels. The collected AFM images were further processed and analyzed using WSxM version 4 develop 11.6 software. ${ }^{60}$

\section{Results and Discussion}

\section{$3.1 \kappa$-Casein aggregation under non-reducing and reducing conditions}

Under (native) physiological conditions, $\kappa$-casein exists as collapsed disordered globules. ${ }^{57}$ In order to follow the aggregation of $\kappa$-casein, ThT fluorescence assay was used. Since $\kappa$-casein contains two cysteine residues that can potentially form intra- and inter-molecular disulfides, we carried out aggregation reaction using a high concentration of $\kappa$-casein $(200 \mu \mathrm{M})$, under reducing (using DTT as a reducing agent) as well as under nonreducing conditions, to decipher the role of disulfides in $\mathrm{K}$-casein aggregation. A time-dependent increase in ThT fluorescence with a typical initial lag phase, was observed with the reduced form of $\kappa$-casein, which is in line with our previous study with RCM $\kappa$-casein ${ }^{61}$ (Figure 1A). However, in the absence of reducing agent, no time-dependent change (for $>48 \mathrm{~h}$ ) in ThT fluorescence was observed which suggests that intermolecular disulfide bonding prevents or slows down $\mathrm{\kappa}$-casein fibrillation (data not shown). This observation is in agreement with previous studies on $\kappa$-casein fibrillation. ${ }^{47}$ However, the aggregation kinetics observed by us for RCM $\kappa$-casein is different from previous studies that showed the absence of lag phase. ${ }^{47,62}$ This discrepancy could arise due to the early oligomer formation when reaction is initiated from RCM $\kappa$-casein in native buffer. ${ }^{47,62}$ In our case, we initiated the aggregation reaction by transfering the protein from the denatured state to the native buffer that 
minimizes the formation of early oligomers. Therefore, in our case the aggregation reaction proceeeded via a nucleation-dependent kinetics comprising a lag phase and an assembly phase.

\subsection{Mechanism of $\kappa$-casein aggregation using multiple fluorescence probes}

Next, we followed the aggregation kinetics using multiple fluorescent probes to discern the molecular events involved in the aggregation process. Fluorescence spectroscopy being a highly sensitive and multi-parametric technique is extensively used for studying protein aggregation. ${ }^{63}$ The steady-state fluorescence spectrum provides insights into the local environment around the fluorophore and information about an overall size of the protein can be obtained from steady state fluorescence anisotropy measurements. ${ }^{63}$ In order to probe the conformational changes taking place during aggregation, we took advantage of the fact that $\kappa$-casein has two cysteines and a single Trp residue. We used Trp as intrinsic fluorophore and AEDANS as extrinsic fluorophore by labeling the thiols of Cys residues with IAEDANS. Additionally, we used ANS, a hydrophobic environment sensitive dye, to follow the aggregation process. The free form of ANS is almost non-fluorescent in water with an emission maximum at $\sim 515 \mathrm{~nm}$. However, it becomes fluorescent upon binding to hydrophobic pockets and undergoes a significant blue shift to $\sim 475 \mathrm{~nm}$ which is mostly accompanied by a rise in intensity. ${ }^{64}$ Due to the ability of ANS to bind to hydrophobic regions/clusters, it is often used in aggregation studies to follow the initial oligomerization and growth steps during fibrillation. All the probes showed the existence of a nucleation-dependent amyloid assembly pathway with a characteristic lag phase, similar to the ThT fluorescence kinetics. The lag time and the rate constants for the aggregation reaction were recovered from the time-dependent plots of all the fluorescence readouts using a previously described relationship ${ }^{59}$
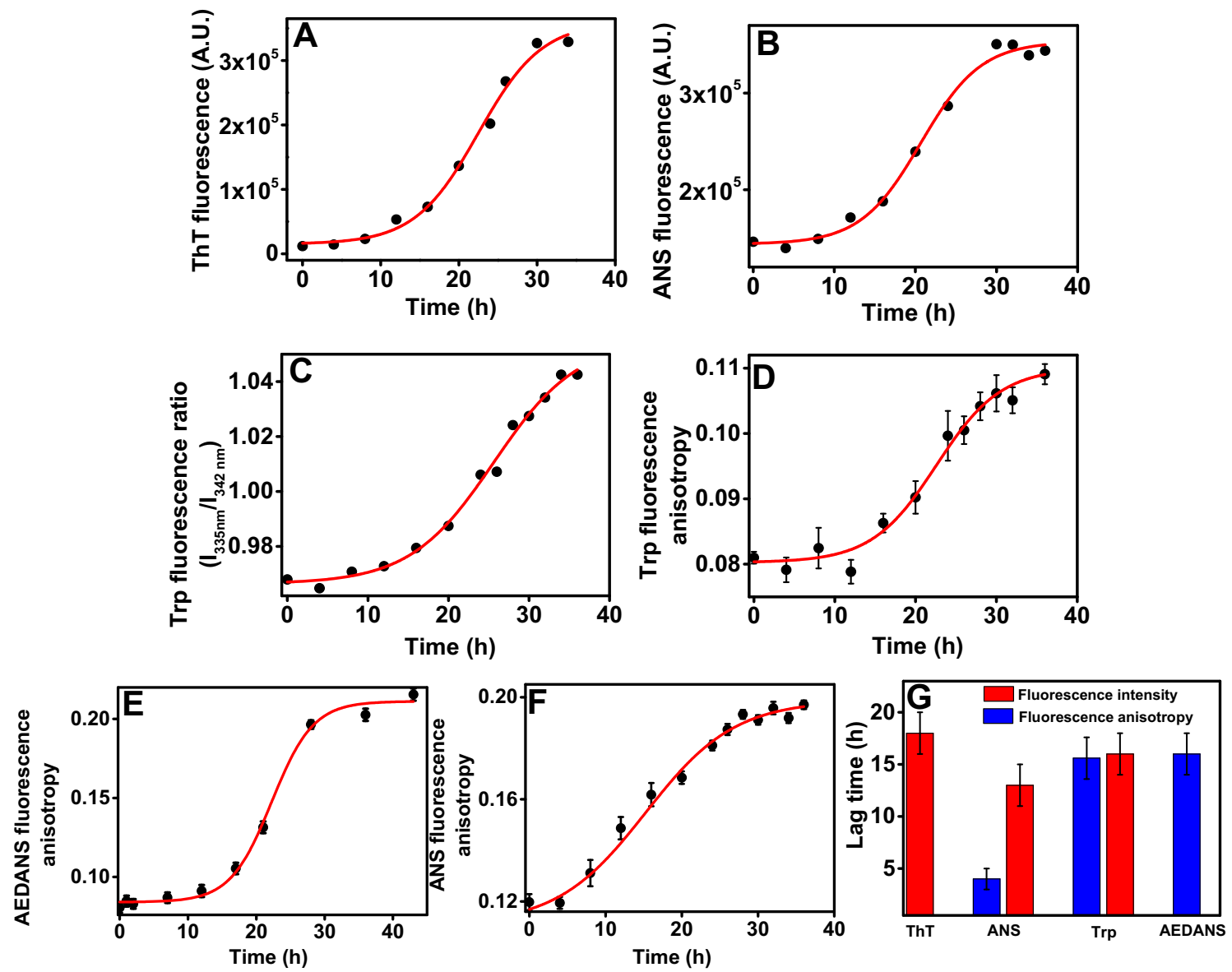

Figure 1. Aggregation kinetics of RCM $\kappa$-casein $(200 \mu \mathrm{M})$ monitored using, (A) ThT fluorescence, (B) ANS fluorescence, (C) Ratio of Trp fluorescence intensity at $335 \mathrm{~nm}$ and $342 \mathrm{~nm}$, (D) Trp fluorescence anisotropy, (E) AEDANS fluorescence anisotropy, and (F) ANS fluorescence anisotropy. (G) The lag time recovered from fitting the time-dependent fluorescence data shown in (A-F) using equation 2 (For details, see Section 2). All the measurements were made at room temperature. 
(Figure 1). The lag time observed for all the fluorescence readouts was found to be comparable, except for the ANS fluorescence anisotropy. Additionally, the growth phase kinetics was found to be similar for all the fluorescence readouts, except for ANS anisotropy. The similarity in the rates indicate that the structural reorganization, particularly in the region harboring Trp and Cys, and aggregation occur simultaneously. The kinetics of the increase in the ANS fluorescence anisotropy was faster during amyloid formation. The monomeric form of $\kappa$-casein exists as collapsed disordered globules ${ }^{57}$ and hence has a very weak affinity for ANS. However, as these collapsed globules coalesce during aggregation process, an increase in ANS fluorescence anisotropy as well as in fluorescence intensity with a concomitant blue shift was observed. The shorter lag phase observed for ANS anisotropy is suggestive of early oligomerization. After characterizing the kinetics of $\kappa$-casein aggregation under physiological conditions, we next embarked upon studies aimed at delineating the effect of SDS on the aggregation process.

\section{$3.3 \kappa$-Casein binds to SDS in a} concentration-dependent manner

We followed the SDS-induced conformational changes in $\kappa$-casein, using $\mathrm{CD}$ and steady-state fluorescence. Upon changing the concentration of SDS, the changes in both $\mathrm{CD}$ and fluorescence readouts were observed. We observed an increase in the ellipticity at $218 \mathrm{~nm}$ as well as $222 \mathrm{~nm}$ with respect to ellipticity at $205 \mathrm{~nm}$, with the increase in SDS concentration (Figure 2A and B). The CD signals at $205 \mathrm{~nm}, 218 \mathrm{~nm}$ and $222 \mathrm{~nm}$ predominantly correspond to random coil, $\beta$-sheets and $\alpha$-helices, respectively. Thus, our CD data indicates that in the presence of SDS, formation of secondary structure at the expense of random coil (Figure 2A and B). It is important to emphasize here that the concentrations of SDS that we have used in our experiments are nondenaturing below the critical micellar concentration of SDS. However, there could be formation of some premicellar aggregates under this condition.

Next, we monitored the changes in the fluorescence attributes of Trp which is present in $\mathrm{Q} / \mathrm{N}$-rich positively
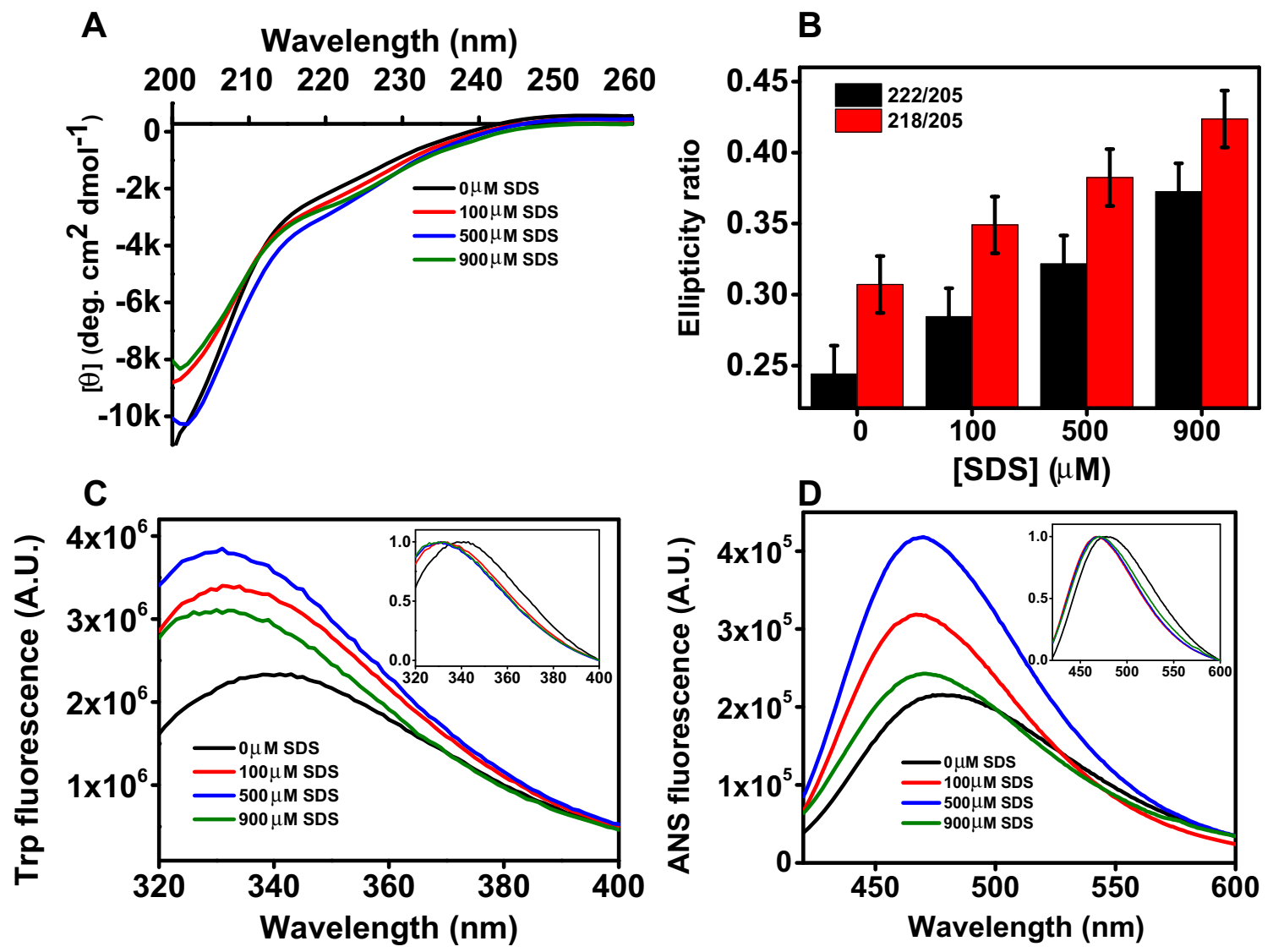

Figure 2. (A) CD spectra for $\kappa$-casein with various concentration of SDS: monomer (black), $100 \mu \mathrm{M}$ (red), $500 \mu \mathrm{M}$ (blue) and $900 \mu \mathrm{M}$ (olive). (B) The ellipticity ratio from CD data are shown in panel A. (C) Trp and (D) ANS fluorescence spectra with color scheme same as in panel A. The normalized Trp and ANS fluorescence spectra are shown as insets to $\mathrm{C}$ and $\mathrm{D}$, respectively. All the measurements were made at room temperature. 

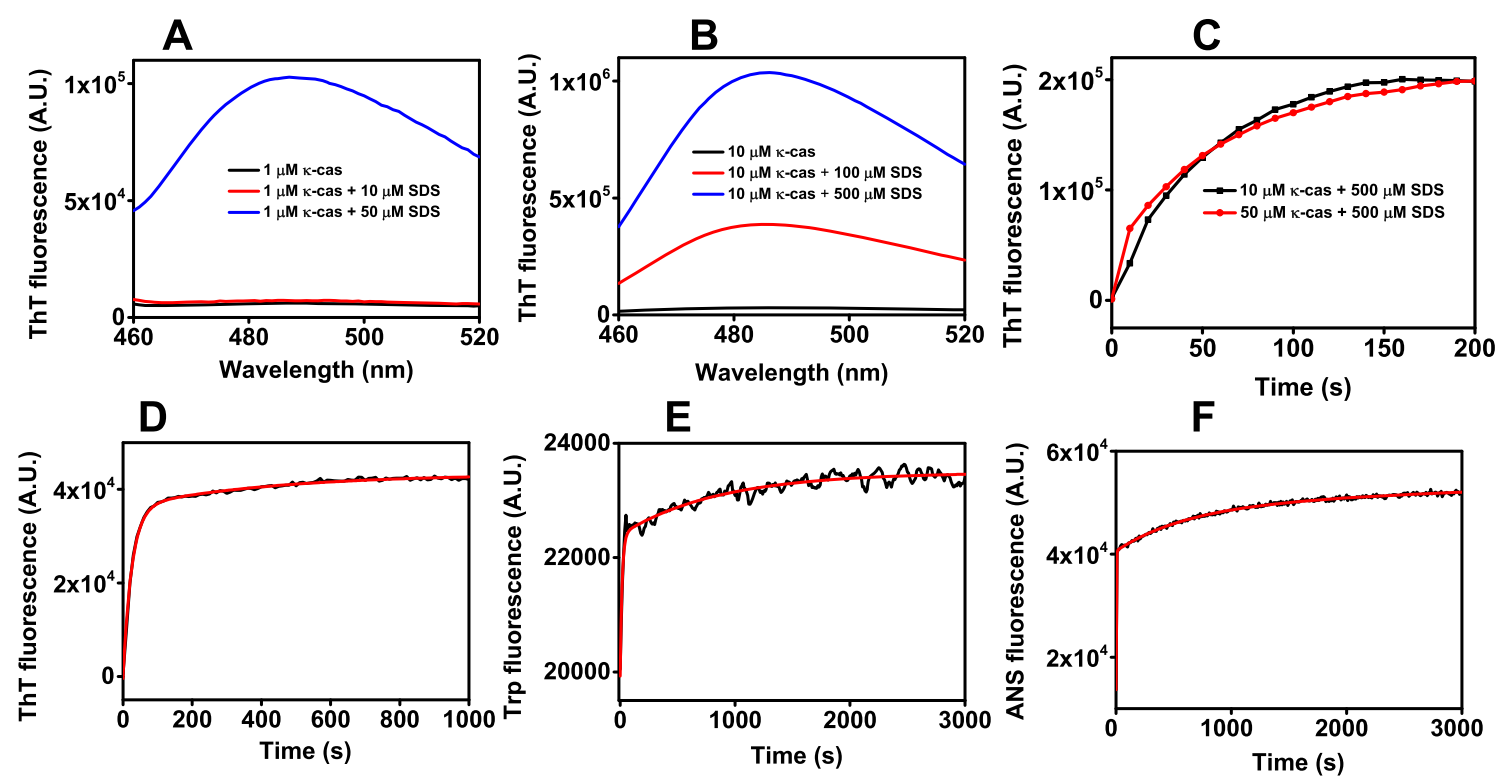

Figure 3. ThT fluorescence spectra for (A) $1 \mu \mathrm{M}$ of $\kappa$-casein without (black) and with (i) $10 \mu \mathrm{M}$ (red) and (ii) $50 \mu \mathrm{M}$ (blue) of SDS. (B) $10 \mu \mathrm{M}$ of $\kappa$-casein without (black) and with (i) $100 \mu \mathrm{M}$ (red) and (ii) $500 \mu \mathrm{M}$ (blue) of SDS. (C) ThT fluorescence kinetics data for (i) $10 \mu \mathrm{M}$ (black) and (ii) $50 \mu \mathrm{M}$ (red) of $\kappa$-casein with $500 \mu \mathrm{M}$ of SDS. (D) ThT, (E) Trp and (F) ANS fluorescence kinetics data for $20 \mu \mathrm{M}$ of $\kappa$-casein with $100 \mu \mathrm{M}$ of SDS. All the measurements were made at room temperature.

charged N-terminal domain. The Q/N-rich domain of $\kappa$-casein shares some resemblance with the amyloidogenic segment of the yeast prion protein. ${ }^{65,66}$ An increase in Trp fluorescence with a concomitant blue shift in the emission maximum was observed with increase in SDS concentration (Figure 2C). At higher concentration of SDS $(>500 \mu \mathrm{M})$, a drop in the fluorescence was observed. However, this drop in the fluorescence was not associated with a red shift in the emission maximum and thus, it is likely to be due to fluorescence quenching from the neighboring amino acid residues. We next monitored the changes in the hydrophobicity. An increase in the ANS fluorescence, accompanied by a blue shift in the emission maximum was observed with increase in SDS concentration and like Trp, at higher concentration of SDS $(>500 \mu \mathrm{M})$, a drop in ANS fluorescence was observed. This set of data suggested that the SDS-induced conformations have higher affinity towards ANS compared to the monomeric state. Since ANS gives us information about the overall hydrophobicity, we speculate that in the presence of SDS, there is formation of some hydrophobic clusters where ANS gets encapsulated. ANS molecules can potentially bind to SDS molecules. However, at the concentrations of SDS that we have used in our study, we did not observe any ANS binding (data not shown).

Taken together, our data from CD and fluorescence suggest that $\kappa$-casein undergoes appreciable conformational change in the presence of SDS. This change appears to be SDS concentration dependent. Though both CD and fluorescence clearly indicated some conformational change in $\kappa$-casein in the presence of SDS, it was not clear whether $\kappa$-casein remains its monomeric state or forms higher order aggregates. Since CD data indicated an increase in $\theta_{218} / \theta_{205}$, we suspected the possibility of aggregation leading to $\beta$-sheet rich conformations in the presence of SDS since SDSinduced fibrillation has been previously reported for some IDPs. ${ }^{33,34,67}$ Therefore, we next carried out ThT fluorescence measurements.

\section{$3.4 \kappa$-Casein aggregates without a lag phase in the presence of $S D S$}

A high affinity for ThT was observed for SDS-induced conformations of $\kappa$-casein and this binding affinity for ThT was found to be highly concentration dependent (Figure 3). The strong dependence on the concentration of $\kappa$-casein as well as SDS was observed (Figure 3A and B). Thus, $\kappa$-casein forms ThT active aggregates in the presence of SDS. In order to decipher the mechanism of SDS-induced aggregation, we followed the kinetics of this conformational change using ThT, Trp and ANS fluorescence. All fluorescent readouts indicated the absence of a lag phase suggesting nucleationindependent or isodesmic polymerization mechanism (Figure 3D-F). ${ }^{68}$ This is clearly in sharp contrast to the nucleation-dependent amyloid assembly kinetics observed for $\kappa$-casein fibrillation in the absence of SDS (Figure 2). Thus, our results suggested that SDS acts as 
A
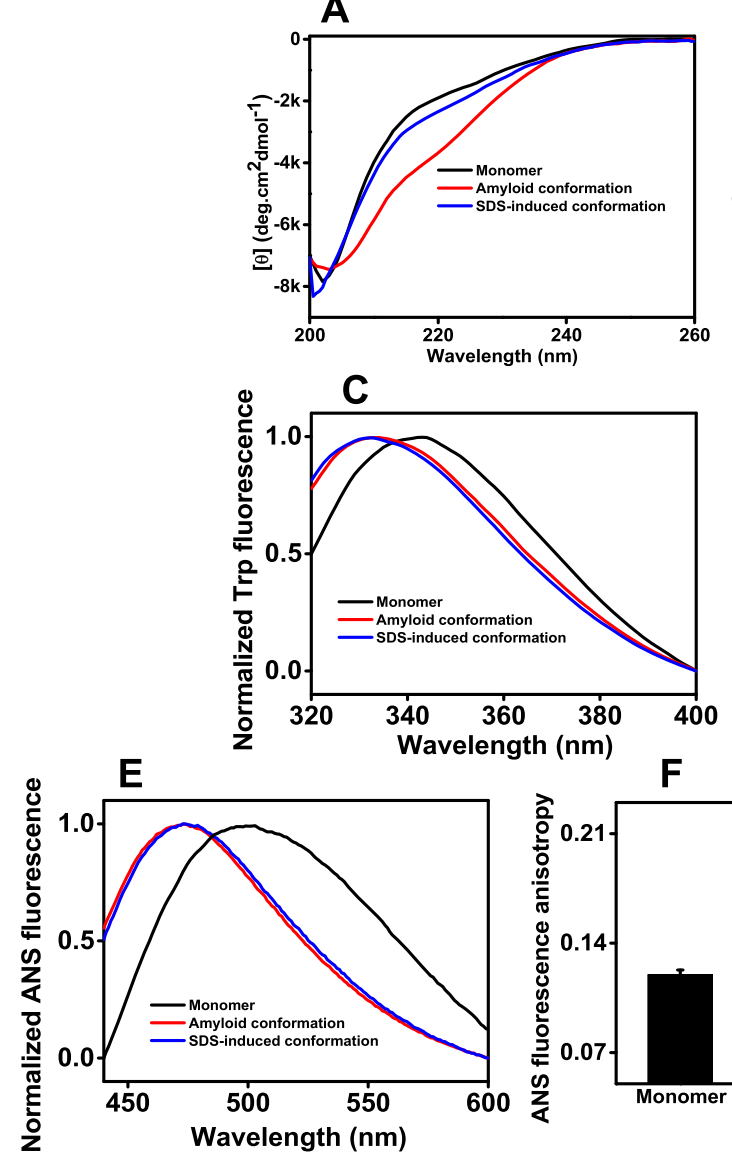

B

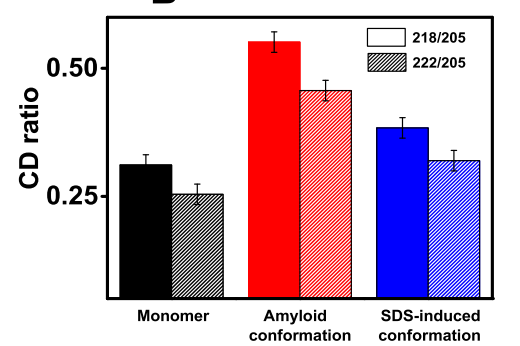

D

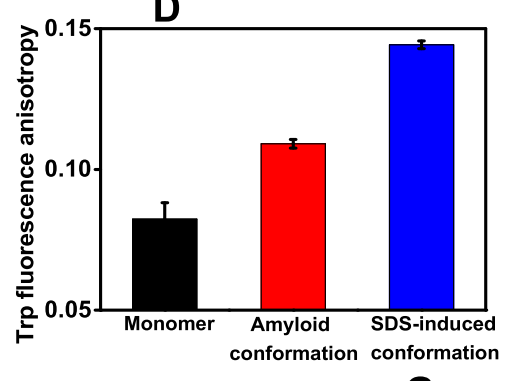

$F$

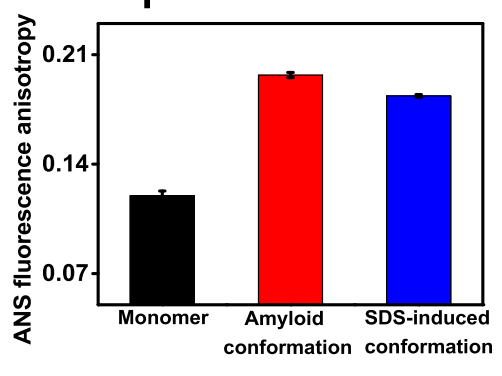

G

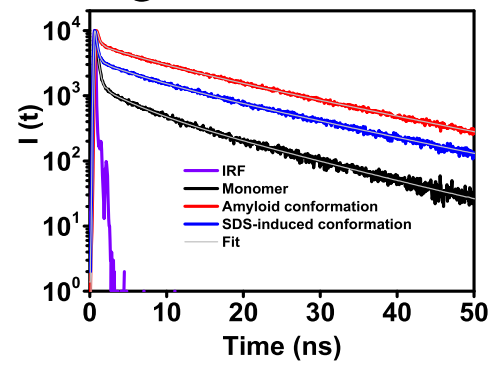

Figure 4. (A) CD spectra, (B) the ellipticity ratio obtained from CD data shown in panel A, (C) Normalized Trp fluorescence spectra, (D) Trp fluorescence anisotropy, (E) Normalized ANS fluorescence spectra, (F) ANS fluorescence anisotropy and (G) ANS fluorescence lifetime for monomer (black), Amyloid conformation (red) and SDS (100 $\mu \mathrm{M})$-induced conformation (blue). All the measurements were made at room temperature.

a trigger for $\kappa$-casein conformational change and aggregation. SDS switches the aggregation mechanism from a typical nucleation dependent polymerization ${ }^{65,68,69}$ to an apparent isodesmic polymerization that does not require a high protein concentration. A similar switch in the aggregation mechanism has been reported previously in the presence of salt and chemical chaperones, upon changing the $\mathrm{pH}$ of the solution, etc. ${ }^{70-72}$

After confirming that $\mathrm{K}$-casein aggregates in the presence of SDS, we next compared the secondary structural contents and fluorescence attributes of the final conformation attained by the aggregates formed in the absence and in the presence of SDS. We refer to the aggregates formed under physiological conditions, in the absence of SDS, as amyloids as it has already been established previously by us that $\kappa$-casein forms amyloid fibrils under this condition. ${ }^{61}$ The aggregate conformation obtained in the presence of SDS has been referred to as SDSinduced conformation. For comparison, we are only considering the aggregates formed with $100 \mu \mathrm{M}$ SDS because the aggregates formed at higher concentration of SDS tend to precipitate as a function of time. It is interesting to note that $\beta$-sheet content is higher for aggregates formed in the absence of SDS as is evident from $\theta_{218} / \theta_{205}$ (Figure 4A and B). In case of Trp fluorescence, though the extent of blue shift is similar for both type of aggregates, anisotropy is much higher for SDSinduced conformation of aggregates (Figure 4C and D). This result indicates that the local structure around Trp is much more rigid in case of aggregates formed in the presence of SDS. This plausibly suggests that SDS neutralize the positive charge in $\mathrm{N}$-terminal domain that harbors Trp and is also rich in aggregation prone $\mathrm{Q}$ and $\mathrm{N}$ residues. The charge neutralization in the $\mathrm{N}$-terminal domain might lead to favorable chain-chain interactions as a result of which aggregation process is accelerated. After Trp, we next compared ANS fluorescence characteristics of both the aggregates. The aggregates formed in the presence of SDS showed much lower ANS binding as is clear from the fluorescence anisotropy and lifetime data (Figure 4E-G). The lower ANS binding for the aggregates formed in the presence of SDS is probably 
associated with the lower content of hydrophobic pockets compared to the aggregates formed without SDS. After gaining insights into the conformational attributes of the aggregates formed under two different solution conditions, we next performed atomic force microscopy (AFM) imaging to visualize the nanoscale morphology of these aggregates.

\subsection{Insights into the nanoscale morphology of aggregates}

AFM is used extensively for imaging the nanoscale topography of protein aggregates. ${ }^{58,73-76}$ In the absence of SDS, we observed long thread-like amyloid fibrils of 6-7 nm in height. The height profiles of these fibrils observed in AFM images collected at different time points in the growth phase of aggregation are shown in Figure 5A-C. In the presence of SDS, AFM imaging revealed a heterogeneous co-existence of small and large-sized spherical oligomers (Figure 5D-F). Mostly, large sized oligomers ranging from $20-60 \mathrm{~nm}$ were observed. Though we could not detect fibrils from these oligomeric species, we do not completely rule out the possibility of fibril formation upon much longer incubation. Previous studies on $\kappa$-casein aggregation have proposed that the rate determining step in $\kappa$-casein fibrillation is the dissociation of larger oligomers into smaller species. ${ }^{48}$ Since in the presence of SDS, mostly large oligomers were observed, it is possible that these oligomers require a much longer time to dissociate into smaller amyloidogenic species. As a result, fibril formation does not occur immediately or within few days. On the contrary, in the absence of SDS, since the oligomers formed are much smaller in size (3-6 nm) and are possibly much more amyloidogenic than the larger species, the fibril formation is much more facile.

Taken together, our studies indicate that in the absence of SDS, $\kappa$-casein aggregation occurs at a high concentration and follows a nucleation-dependent polymerization pathway. In the presence of SDS, a switch in the aggregation mechanism from nucleation-dependent polymerization to an apparent isodesmic polymerization is observed. The alteration in the aggregation mechanism also leads to a variation in the morphology of the aggregates suggesting that the morphology of the protein aggregates might be strongly linked to the
A

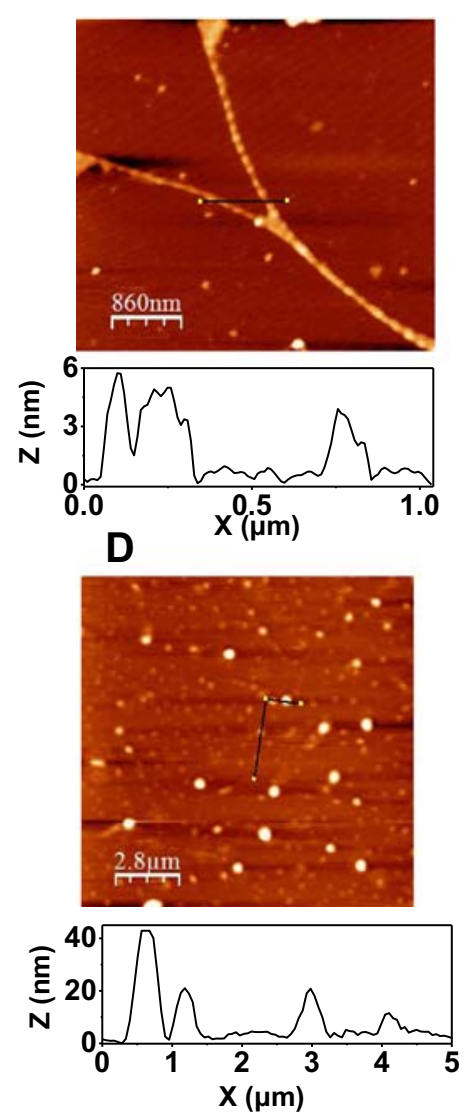

B
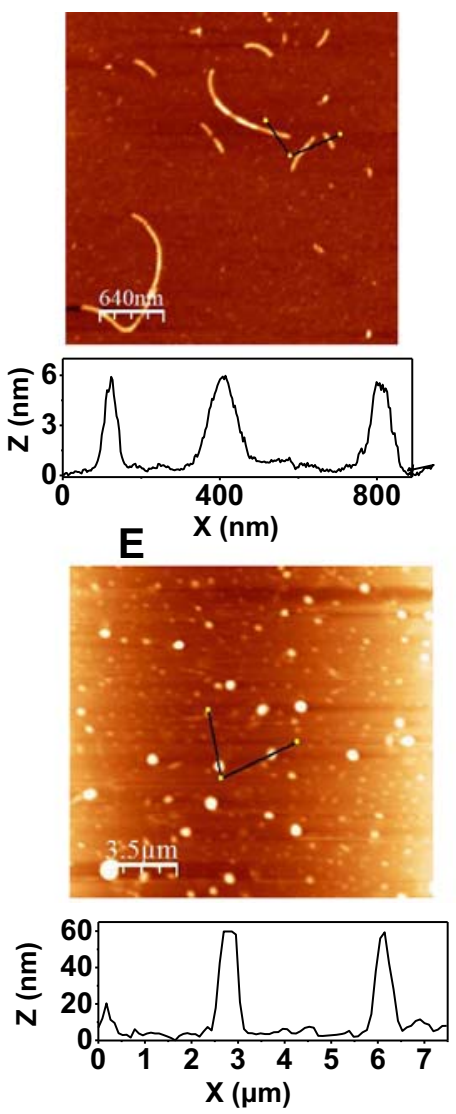

C
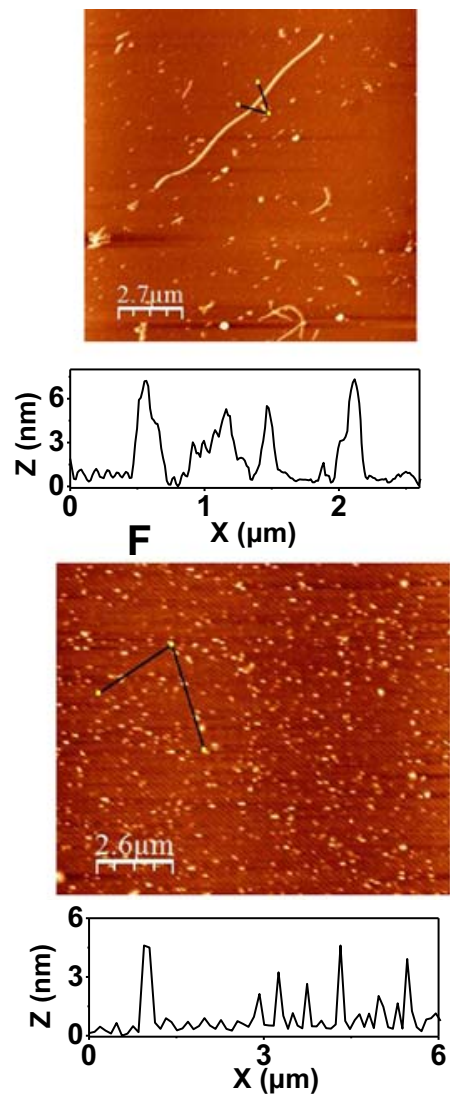

Figure 5. AFM images along with the height profiles of $\kappa$-casein $(A-C)$ amyloid fibrils formed without SDS and (D-F) spherical oligomeric aggregates formed in the presence of SDS (100 $\mu \mathrm{M})$. 
mechanism of aggregation. We believe that SDS acts as a conformational catalyst by neutralizing the positive charge on the aggregation prone $\mathrm{Q} / \mathrm{N}$ rich domain that results in the hydrophobic association and intermolecular hydrogen bonding between the polypeptide chains. This in turn facilitates the coalescence of $\kappa$ casein monomers into large sized oligomers and is in line with the charge colloid model. ${ }^{77,78}$ As a result of facile oligomerization due to charge neutralization in the presence of anionic SDS, the lag phase is not detected during aggregation in the presence of SDS.

\section{Conclusions}

In this work, we showed the effect of a well-known lipid mimetic, SDS, on the mechanism of aggregation of an amyloidogenic IDP, namely, bovine $\kappa$-casein using a variety of biophysical tools. The deposition of amyloids formed by $\kappa$-casein is linked with mammary cancer. The amyloid formation from $\kappa$-casein followed a nucleation dependent polymerization in the absence of SDS, as shown previously. ${ }^{61}$ In the presence of SDS at submicellar concentrations, a switch in the mechanism was observed. Additionally, the morphologies of the aggregates formed in the absence and presence of SDS were found to be different. Without SDS, fibril formation occurred, whereas, in the presence of SDS, mostly large spherical oligomers were observed. The fibrillar species formed without SDS have higher $\beta$-sheet content compared to the spherical oligomeric species formed in the presence of SDS. The interaction of $\kappa$-casein with SDS may have physiological relevance as SDS being a membrane mimetic is able to mimic the role of anionic lipids found in milk and milk ducts. Additionally, our studies suggest that the morphology of the protein aggregates can be modulated using detergents and can be utilized for the designing of novel protein/amyloid based nanomaterials. Since $\kappa$-casein is readily available and easy to isolate from milk, therefore, by making use of lipid mimetics/surfactants, we can control the aggregation pathway of $\kappa$-casein, modulate the morphology of the aggregates and accordingly utilize them for various bionanomaterials applications. In fact, the fibrillar $\kappa$-casein has been used in the past for the encapsulation and controlled delivery of retinoic acid, which was used to promote the differentiation of neuronal cells. ${ }^{79}$

\section{Acknowledgements}

We thank IISER Mohali, the Council of Scientific and Industrial Research, the Department of Science and Technology, the Ministry of Human Resource Development, Govt. of India (research grants to S.M. and fellowships to S.A., P.D. \& N.J.) for financial support.

\section{References}

1. Nelson R, Sawaya M R, Balbirnie M, Madsen A O, Riekel C, Grothe R and Eisenberg D 2005 Structure of the cross-[beta] spine of amyloid-like fibrils Nature $\mathbf{4 3 5}$ 773

2. Brettschneider J, Tredici K D, Lee V M Y and Trojanowski J Q 2015 Spreading of pathology in neurodegenerative diseases: A focus on human studies Nat. Rev. Neurosci. 16109

3. Uversky V N, Dave V, Iakoucheva L M, Malaney P, Metallo S J, Pathak R R and Joerger A C 2014 Pathological unfoldomics of uncontrolled chaos: Intrinsically disordered proteins and human diseases Chem. Rev. 1146844

4. Tipping K W, van Oosten-Hawle P, Hewitt E W and Radford S E 2015 Amyloid fibres: Inert end-stage aggregates or key players in disease? Trends Biochem. Sci. 40719

5. Knowles T P, Vendruscolo M and Dobson C M 2014 The amyloid state and its association with protein misfolding diseases Nat. Rev. Mol. Cell Biol. 15384

6. Eisenberg D and Jucker M 2012 The amyloid state of proteins in human diseases Cell 1481188

7. Greenwald J and Riek R 2010 Biology of amyloid: Structure, function, and regulation Structure 181244

8. LiC and Mezzenga R 2013 The interplay between carbon nanomaterials and amyloid fibrils in bio-nanotechnology Nanoscale 56207

9. Bolisetty S and Mezzenga R 2016 Amyloid-carbon hybrid membranes for universal water purification Nat. Nanotechnol. 11365

10. Knowles T P and Mezzenga R 2016 Amyloid fibrils as building blocks for natural and artificial functional materials Adv. Mater. 286546

11. Cherny I and Gazit E 2008 Amyloids: Not only pathological agents but also ordered nanomaterials Angew. Chem. Int. Ed. 474062

12. Pearce F G, Mackintosh S H and Gerrard J A 2007 Formation of amyloid-like fibrils by ovalbumin and related proteins under conditions relevant to food processing $J$. Agric. Food Chem. $\mathbf{5 5} 318$

13. Raynes J K, Carver J A, Gras S L and Gerrard J A 2014 Protein nanostructures in food-Should we be worried? Trends Food Sci. Technol. 3742

14. Stefani M and Dobson C M 2003 Protein aggregation and aggregate toxicity: New insights into protein folding, misfolding diseases and biological evolution J. Mol. Med. 81678

15. Cohen S I A, Vendruscolo M, Dobson C M and Knowles T P 2012 From macroscopic measurements to microscopic mechanisms of protein aggregation J. Mol. Biol. 421160

16. Knowles T P, Waudby C A, Devlin G L, Cohen S I A, Aguzzi A, Vendruscolo M, Terentjev E M, Welland M E and Dobson C M 2009 An analytical solution to the kinetics of breakable filament assembly Science $\mathbf{3 2 6} 1533$

17. Bucciantini M, Rigacci S and Stefani M 2014 Amyloid aggregation: Role of biological membranes and the aggregate-membrane system J. Phys. Chem. Lett. 5517 
18. Bucciantini M and Cecchi C 2010 Biological membranes as protein aggregation matrices and targets of amyloid toxicity. In Protein Misfolding and Cellular Stress in Disease and Aging: Concepts and Protocols $\mathrm{P}$ Bross and $\mathrm{N}$ Gregersen (Eds.) (Totowa, NJ: Humana Press) p. 231

19. Sethuraman A and Belfort G 2005 Protein structural perturbation and aggregation on homogeneous surfaces Biophys. J. 881322

20. Zhu J, Nathan C, Jin W, Sim D, Ashcroft G S, Wahl S M, Lacomis L, Erdjument-Bromage H, Tempst P, Wright C D and Ding A 2002 Conversion of proepithelin to epithelins: Roles of SLPI and elastase in host defense and wound repair Cell 111867

21. Gorbenko G P and Kinnunen P K J 2006 The role of lipid-protein interactions in amyloid-type protein fibril formation Chem. Phys. Lipids 14172

22. Michikawa M, Gong J S, Fan Q W, Sawamura N and Yanagisawa K 2001 A novel action of Alzheimer's amyloid $\beta$-protein $(\mathrm{A} \beta)$ : Oligomeric $\mathrm{A} \beta$ promotes lipid release J. Neurosci. 217226

23. Sparr E, Engel M F M, Sakharov D V, Sprong M, Jacobs J, de Kruijff B, Höppener J W M and Antoinette Killian J 2004 Islet amyloid polypeptide-induced membrane leakage involves uptake of lipids by forming amyloid fibers FEBS Lett. 577117

24. Zhu M, Li J and Fink A L 2003 The association of $\alpha$-synuclein with membranes affects bilayer structure, stability, and fibril formation J. Biol. Chem. 27840186

25. Chaudhary H, Stefanovic A N D, Subramaniam V and Claessens M M A E 2014 Membrane interactions and fibrillization of $\alpha$-synuclein play an essential role in membrane disruption FEBS Lett. $\mathbf{5 8 8} 4457$

26. Reynolds N P, Soragni A, Rabe M, Verdes D, Liverani E, Handschin S, Riek R and Seeger S 2011 Mechanism of membrane interaction and disruption by $\alpha$-synuclein J. Am. Chem. Soc. 13319366

27. Lashuel H A 2005 Membrane permeabilization: A common mechanism in protein-misfolding diseases $\mathrm{Sci}$. Aging Knowledge Environ. 2005 pe28

28. Cao P, Abedini A, Wang H, Tu L-H, Zhang X, Schmidt A M and Raleigh D P 2013 Islet amyloid polypeptide toxicity and membrane interactions Proc. Natl. Acad. Sci. USA 11019279

29. Goodchild S C, Sheynis T, Thompson R, Tipping K W, Xue W F, Ranson N A, Beales P A, Hewitt E W and Radford S E 2014 ß2-Microglobulin amyloid fibril-induced membrane disruption is enhanced by endosomal lipids and acidic $\mathrm{pH} P$ LOS ONE 9 e 104492

30. Milanesi L, Sheynis T, Xue W-F, Orlova E V, Hellewell A L, Jelinek R, Hewitt E W, Radford S E and Saibil H R 2012 Direct three-dimensional visualization of membrane disruption by amyloid fibrils Proc. Natl. Acad. Sci. USA 10920455

31. van der Lee R, Buljan M, Lang B, Weatheritt R J, Daughdrill G W, Dunker A K, Fuxreiter M, Gough J, Gsponer J, Jones D T, Kim P M, Kriwacki R W, Oldfield C J, Pappu R V, Tompa P, Uversky V N, Wright P E and Babu M M 2014 Classification of intrinsically disordered regions and proteins Chem. Rev. 1146589

32. Dyson H J and Wright P E 2005 Intrinsically unstructured proteins and their functions Nat. Rev. Mol. Cell Biol. 6197
33. Rivers R C, Kumita J R, Tartaglia G G, Dedmon M M, Pawar A, Vendruscolo M, Dobson C M and Christodoulou J 2008 Molecular determinants of the aggregation behavior of $\alpha$ - and $\beta$-synuclein Protein Sci. 17887

34. Giehm L, Oliveira C L P, Christiansen G, Pedersen J S and Otzen D E 2010 SDS-induced fibrillation of $\alpha$ synuclein: An alternative fibrillation pathway $\mathrm{J}$. Mol. Biol. 401115

35. Ferreon A C, Gambin Y, Lemke E A and Deniz A A 2009 Interplay of alpha-synuclein binding and conformational switching probed by single-molecule fluorescence Proc. Natl. Acad. Sci. USA 1065645

36. Ahmad M F, Ramakrishna T, Raman B and Rao Ch M 2006 Fibrillogenic and non-fibrillogenic ensembles of SDS-bound human alpha-synuclein J. Mol. Biol. 364 1061

37. Tompa P and Fersht 2009 In A Structure and Function of Intrinsically Disordered Proteins (Florida: Taylor \& Francis Group)

38. Farrell H M, Kumosinski T F, Malin E L and Brown E M 2002 The caseins of milk as calcium-binding proteins. In Calcium-Binding Protein Protocols: Volume 1: Reviews and Case Studies H J Vogel (Ed.) (Totowa, NJ: Humana Press) p. 97

39. De Kruif C G and Holt C 2003 Casein micelle structure, functions and interactions. In Advanced Dairy Chemistry-1 Proteins: Part A/Part B P F Fox and P L H McSweeney (Eds.) (Boston, MA: Springer) p. 233

40. Bhattacharyya J and Das K P 1999 Molecular chaperonelike properties of an unfolded protein, as-casein J. Biol. Chem. 27415505

41. Zhang X, Fu X, Zhang H, Liu C, Jiao W and Chang Z 2005 Chaperone-like activity of $\beta$-casein Int. J. Biochem. Cell Biol. 371232

42. Morgan P E, Treweek T M, Lindner R A, Price W E and Carver J A 2005 Casein proteins as molecular chaperones J. Agric. Food Chem. $\mathbf{5 3} 2670$

43. Yong Y H and Foegeding E A 2010 Caseins: Utilizing molecular chaperone properties to control protein aggregation in foods J. Agric. Food Chem. $\mathbf{5 8} 685$

44. Holt C, Carver J A, Ecroyd H and Thorn D C 2013 Invited review: Caseins and the casein micelle: Their biological functions, structures, and behavior in foods J. Dairy Sci. 966127

45. Farrell H M, Cooke P H, Wickham E D, Piotrowski E G and Hoagland P D 2003 Environmental influences on bovine $\kappa$-casein: Reduction and conversion to fibrillar (amyloid) structures J. Protein Chem. 22259

46. Thorn D C, Ecroyd H, Sunde M, Poon S and Carver J A 2008 Amyloid fibril formation by bovine milk $\alpha$ s2-casein occurs under physiological conditions yet is prevented by its natural counterpart, as1-casein Biochemistry $\mathbf{4 7} 3926$

47. Thorn D C, Meehan S, Sunde M, Rekas A, Gras S L, MacPhee C E, Dobson C M, Wilson M R and Carver J A 2005 Amyloid fibril formation by bovine milk k-casein and its inhibition by the molecular chaperones $\alpha \mathrm{S}$ - and $\beta$-casein Biochemistry $\mathbf{4 4} 17027$

48. Ecroyd H, Thorn David C, Liu Y and Carver John A 2010 The dissociated form of $\kappa$-casein is the pre- 
cursor to its amyloid fibril formation Biochem. J. 429 251

49. Sokolovski M, Sheynis T, Kolusheva S and Jelinek R 2008 Membrane interactions and lipid binding of casein oligomers and early aggregates Biochim. Biophys. Acta 17782341

50. MacGibbon A K H and Taylor M W 2006 Composition and structure of bovine milk lipids. In Advanced Dairy Chemistry Volume 2 Lipids P F Fox and P L H McSweeney (Eds.) (Boston, MA: Springer) p. 1

51. Liu J, Dehle F C, Liu Y, Bahraminejad E, Ecroyd H, Thorn D C and Carver J A 2016 The effect of milk constituents and crowding agents on amyloid fibril formation by к-casein J. Agric. Food Chem. 641335

52. Sacchettini J C and Kelly J W 2002 Therapeutic strategies for human amyloid diseases Nat. Rev. Drug Discov. 1267

53. Rhie A, Kirby L, Sayer N, Wellesley R, Disterer P, Sylvester I, Gill A, Hope J, James W and Tahiri-Alaoui A 2003 Characterization of $2^{\prime}$-fluoro-RNA aptamers that bind preferentially to disease-associated conformations of prion protein and inhibit conversion J. Biol. Chem. 27839697

54. Ryan T M, Griffin M D W, Teoh C L, Ooi J and Howlett G J 2011 High-affinity amphipathic modulators of amyloid fibril nucleation and elongation J. Mol. Biol. 406416

55. Ryan T M, Friedhuber A, Lind M, Howlett G J, Masters C and Roberts B R 2012 Small amphipathic molecules modulate secondary structure and amyloid fibril-forming kinetics of alzheimer disease peptide A $\beta 1-42 \mathrm{~J}$. Biol. Chem. 28716947

56. Arya S and Mukhopadhyay S 2014 Ordered water within the collapsed globules of an amyloidogenic intrinsically disordered protein J. Phys. Chem. B 1189191

57. Jain N, Bhattacharya M and Mukhopadhyay S 2011 Chain collapse of an amyloidogenic intrinsically disordered protein Biophys. J. 1011720

58. Arya S, Kumari A, Dalal V, Bhattacharya M and Mukhopadhyay S 2015 Appearance of annular ringlike intermediates during amyloid fibril formation from human serum albumin Phys. Chem. Chem. Phys. 17 22862

59. Arora A, Ha C and Park C B 2004 Inhibition of insulin amyloid formation by small stress molecules FEBS Lett. $\mathbf{5 6 4} 121$

60. Horcas I, Fernández R, Gómez-Rodríguez J M, Colchero J, Gómez-Herrero J and Baro A M 2007 WSXM: A software for scanning probe microscopy and a tool for nanotechnology Rev. Sci. Instrum. 78013705

61. Arya S, Singh A K, Khan T, Bhattacharya M, Datta A and Mukhopadhyay S 2016 Water rearrangements upon disorder-to-order amyloid transition J. Phys. Chem. Lett. 74105

62. Ecroyd H, Thorn David C, Liu Y and Carver John A 2010 The dissociated form of $\kappa$-casein is the precursor to its amyloid fibril formation Biochem. J. 429 251

63. Lakowicz J R 2007 In Principles of Fluorescence Spectroscopy J R Lakowicz (Ed.) (New York: Springer)
64. Daniel E and Weber G 1966 Cooperative effects in binding by bovine serum albumin. I. The Binding of 1anilino-8-naphthalenesulfonate. Fluorimetric titrations Biochemistry 51893

65. Krishnan R and Lindquist S L 2005 Structural insights into a yeast prion illuminate nucleation and strain diversity Nature $\mathbf{4 3 5} 765$

66. Mukhopadhyay S, Krishnan R, Lemke E A, Lindquist S and Deniz A A 2007 A natively unfolded yeast prion monomer adopts an ensemble of collapsed and rapidly fluctuating structures Proc. Natl. Acad. Sci. USA 104 2649

67. Abelein A, Kaspersen J D, Nielsen S B, Jensen G V, Christiansen G, Pedersen J S, Danielsson J, Otzen D E and Gräslund A 2013 Formation of dynamic soluble surfactant-induced amyloid $\beta$ peptide aggregation intermediates J. Biol. Chem. 28823518

68. Frieden $\mathrm{C} 2007$ Protein aggregation processes: In search of the mechanism Protein Sci. 162334

69. Lee J, Culyba E K, Powers E T and Kelly J W 2011 Amyloid-beta forms fibrils by nucleated conformational conversion of oligomers Nat. Chem. Biol. 7602

70. Jain S and Udgaonkar J 2010 Salt-induced modulation of the pathway of amyloid fibril formation by the mouse prion protein Biochemistry 497615

71. Borzova V A, Markossian K A, Kleymenov S Y and Kurganov B I 2017 A change in the aggregation pathway of bovine serum albumin in the presence of arginine and its derivatives Sci. Rep. 73984

72. Dogra P, Bhattacharya M and Mukhopadhyay S 2017 $\mathrm{pH}$-Responsive mechanistic switch regulates the formation of dendritic and fibrillar nanostructures of a functional amyloid J. Phys. Chem. B 121412

73. Bhattacharya M, Jain N, Dogra P, Samai S and Mukhopadhyay S 2013 Nanoscopic amyloid pores formed via stepwise protein assembly J. Phys. Chem. Lett. 4480

74. Usov I, Adamcik J and Mezzenga R 2013 Polymorphism in bovine serum albumin fibrils: Morphology and statistical analysis Faraday Discuss. 166151

75. Volpatti L R, Vendruscolo M, Dobson C M and Knowles T P 2013 A clear view of polymorphism, twist, and chirality in amyloid fibril formation ACS Nano 710443

76. Sweers K K M, van der Werf, K O, Bennink M L and Subramaniam V 2012 Atomic force microscopy under controlled conditions reveals structure of C-terminal region of $\alpha$-synuclein in amyloid fibrils ACS Nano 65952

77. Sciortino F, Mossa S, Zaccarelli E and Tartaglia P 2004 Equilibrium cluster phases and low-density arrested disordered states: The role of short-range attraction and long-range repulsion Phys. Rev. Lett. 93055701

78. Sahoo B, Nag S, Sengupta P and Maiti S 2009 On the stability of the soluble amyloid aggregates Biophys. J. 971454

79. Chun J, Bhak G, Lee S G, Lee J H, Lee D, Char K and Paik S R 2012 к-Casein-based hierarchical suprastructures and their use for selective temporal and spatial control over neuronal differentiation Biomacromolecules 132731 